\title{
Power-law Distribution of Family Names in Japanese Societies
}

\author{
Sasuke Miyazima ${ }^{1}$, Youngki Lee ${ }^{2}$, Tomomasa Nagamine ${ }^{1}$ and Hiroaki Miyajima ${ }^{3}$ \\ ${ }^{1}$ Department of Engineering Physics, Chubu University, Kasugai, Aichi 487-8501, Japan \\ ${ }^{2}$ Center for Polymer Studies 83 Department of Physics, Boston University, Boston, MA 02215, \\ $U S A$ \\ ${ }^{3}$ Department of Space Science, Ohio State University, Columbus, OH 43210, USA \\ (Last modified: December 1, 1998; Printed: June 4, 2021)
}

\begin{abstract}
We study the frequency distribution of family names. From a common data base, we count the number of people who share the same family name. This is the size of the family. We find that (i) the total number of different family names in a society scales as a power-law of the population, (ii) the total number of family names of the same size decreases as the size increases with a power-law and (iii) the relation between size and rank of a family name also shows a power-law. These scaling properties are found to be consistent for five different regional communities in Japan.
\end{abstract}

Scaling laws have been playing an important role in science for the past several decades [1]. Diverse systems in nature have been found to exhibit a scaling law and self-similarity without a fine tuning of external parameters — known as self-organized-criticality. A simple model proposed by Bak et. al. [2] shows that the minimal ingredients of these scaling behaviours is to have a large number of degrees of freedom and nonlinear interactions between them. Human societies also show complexity which meets the above features of self-organized criticality. In this context, many of human activities including word freqeuncy [3], traffic 
flow [4], economics [5], population growth [6], city growth [7], internet [8], citation frequency [9] and war distribution [10] have been reported to show scaling behaviour.

Here, we study frequency distribution of family names in Japanese societies. We define a family as a group of people who share the same family name, i.e., the different families are identified by their family names. We also define the size of a family, s, as a number of people in that family. We rank families by their size from the biggest family to the smallest family; For example, the biggest family in town of Fuso is "Senda" with family size $s($ Senda $)=296$, so its rank is $r($ Senda $)=1$. The second biggest is "Kondo" with size $s($ Kondo $)=229$, and $\operatorname{rank} r(K$ ondo $)=2$, and so on [11]. In this way we measure the size and the rank of all families.

We analyze the telephone directories of five regional communities in Japan: town of Haruhi, town of Fuso, city of Inazawa, city of Kasugai and 1/3 of the city of Nagoya. The directories were published in 1998 by the communications company "NTT". The total number of customers $S$ appeared in these directories are 1634, 7775, 23365, 65988 and 177267, respectively. First, we count the number of different family names, $N$, appeared in the directories. In Fig. 11 we plot $N$ versus $S$ and we find that

$$
N \sim S^{\chi}
$$

with an exponent $\chi=0.65 \pm 0.03$.

Next, we investigate the scaling properties of two different quantities: (i) the distribution of the family size $n(s)$ which is the number of families of the same size $s$, and (ii) the relation between size and rank of a family, i.e., $s(r)$ which forms the so-called Zipf's plot [3]. The two quantities are complementary in a sense that $n(s)$ mainly focuses on the scaling property of the smaller size family while $s(r)$ highlights the scaling property of the bigger size family. We find the power-law scalings for both the quantities which are consistent for all five regions investigated.

We measure the distribution $n(s)$ for each town which is shown in Fig. 2a in double logarithmic scale. It shows a nice power-law behaviour with same exponent for all five 
different communities. We suggest the following scaling form for $n(s)$ [12]:

$$
n(s)=A f\left(\frac{s}{s^{*}}\right)
$$

where the scaling function $f(x)$ behaves as $f \sim x^{-\tau}$ for $x \ll 1$ and $f=1$ for $x \gg 1$. Here $s^{*}$ is a characteristic family size at which $n$ becomes one, i.e. $n\left(s^{*}\right)=1$, which in turn gives $A=1$. In Fig. 2b we try to collapse data using the scaling form of Eq. (2) with an additional scaling law $s^{*} \sim S^{\alpha}$ and the scaling exponent $\alpha=0.37 \pm 0.03$. A linear fit of the collapsed scaling function yields $\tau=1.75 \pm 0.05$.

From the normalization condition,

$$
\int_{1}^{s_{*}} n(s) d s=N
$$

and the scaling form for $n(s)$ [Eq. (2)] we obtain a relation, $s^{*} \sim N^{1 / \tau}$. This scaling, combined with our finding $N \sim S^{\chi}$, gives

$$
\alpha=\frac{\chi}{\tau}
$$

This scaling relation is well consistent with the exponents measured within error bars.

In Fig. Ba we plot the family size $s$ versus rank $r$ in double logarithmic scale. Each curve shows a crossover behaviour from one power-law regime with exponent $\phi_{I}=0.67 \pm 0.03$, to another steeper power-law decay with exponent $\phi_{I I}=1.33 \pm 0.03$ at the characteristic rank $r^{*}$ which also scales as $r^{*} \sim S^{\alpha^{\prime}}$. We propose the following scaling form for $s(r)$;

$$
s(r)=r^{*} g\left(\frac{r}{r^{*}}\right)
$$

where the scaling function $g$ behaves as $g \sim x^{-\phi_{I}}$ for $x \ll 1$ and $g \sim x^{-\phi_{I I}}$ for $x \gg 1$. In Fig. 3b we try to collapse the data using the scaling form of Eq. (5) and the best fit is obtained when $\alpha^{\prime}=0.5 \pm 0.05$.

Two quantities, $n(s)$ and $r(s)$, are related by an integral equation [14];

$$
r(s)=\int_{s}^{\infty} n\left(s^{\prime}\right) d s^{\prime} \sim s^{1-\tau}
$$


By inverting the relation we obtain a scaling relation, $s(r) \sim r^{\frac{1}{1-\tau}}$. This relation gives the exponent

$$
\phi_{I I}=\frac{1}{\tau-1}
$$

because the scaling exponent $\tau$ is measured for small $s$, i.e. for $r>r^{*}$. Note that the Eq. (7) is well satisfied by our results. The fact that the crossover points $r^{*}$ scales as $S^{0.5}$ suggests that the sampling of the population is random so that relative deviation of the probability decreases as $S^{-0.5}$ as number of data points $S$ increases.

To test the role of the communities on the observed scaling behaviours we randomly select a population and repeat our analysis for the extracted data set. Figure 4 shows the distributions for the randomly chosen population $S=2189,6566,19696,59089$ and 177267 out of the biggest data for $1 / 3$ of city of Nagaya. It shows very close scaling behaviours as Figs. 11 to 3. This experiment suggests that the families are distributed randomly in the town without spatial correlation. Such scaling universality in the family structure of contemporary societies could be explained as a result that the time scale characterizing the migration of pupulation in a community is much shorter than the time scale asscoiated with the reproduction of a famiy name.

The scaling exponents $\tau=1.75, \phi_{I}=0.67$ and $\phi_{I I}=1.33$ are different from the Zipf's result on word frequency where the exponents are $\tau=2.0$ and $\phi=1.0$. The power-law relation between $N$ and $S$ and it's exponent $\chi=0.65$ observed in family name distribution seem to be nontrivial. One may expect this scaling law breaks if the number of available family names in a society is too small compared to the population. Cohen et. al. [14] found that this situation occurred in the words frequency distribution - for very large $S, N(S)$ approaches a plateau. They found that the exponent $\chi$ for the number of different words in a text is also a function of length of the text. This is true also for the societies where the family names are strictly inherited from fathers to sons without any creation of new family names. In fact, the expectation number of sons per parents is one under the stationary constant population. Then the survival probability $P(t)$ of a family name after $t$ generations decreases 
as $P(t) \sim t^{-0.5}$. As a result, after many generations, only a few family names will dominate the whole population in the society. This is the situation in countries where the creation of new family names has been strictly restricted for many generations such as in Korea. The total number of family names in Korea is about 250 while the total population is about 50 millions. On the contrary Japan has most rich family names in the world whose total number of family names is about 132,000 and the population is about 125 millions. The creation of a new family name in Japan is also very rare. However, historically the most of Japanese family names were created about 120 years ago [15. The short history of family names may cause to preserve the diversity and the scaling properties of family names as it was at the creation.

In summary, we have investigated the distribution of Japanese family names for five different regional communities in Japan. From the our empirical investigation, the powerlaw relation between total number of different family names and total population appeared in a telephone directory with the exponent $\chi=0.65$. Also we have found that the name-varietysize distribution shows nice power-law scaling with the exponent $\tau=1.75$ and the cutoff exponent, $\alpha=0.37$. These scaling properties are consistent for five regional communities and randomly generated societies with with different populations. In a size-rank distribution of family name we have obtained a crossover behaviour from one exponent, $\phi_{I}=0.67$ to another exponent $\phi_{I I}=1.33$ at the crossover point $r^{*} \sim S^{\alpha^{\prime}}$ with $\alpha^{\prime}=0.5$. This result is consistent even if the specific family names of higher rank in one community is different from those in other communities. We have also derived scaling relations between these exponents.

\section{Acknowdgements}

We thank I. Grosse, P.Ch. Ivanov and S. Havlin for helpful discussions. 


\section{REFERENCES}

[1] B. Widom, J. Chem. Phys. 43 (1965) 3892, and a summary of the mean field method can be found in R. Brout, Phase Transitions, W.A. Benjamin Inc., New York, 1965, and C. Domb and M.S. Green, Phase Transitions and Critical Phenomena, vol 5a, Academic Press, London, 1976.

[2] P. Bak, C. Tang and K. Wiesenfeld, Phys. Rev. A 38 (1988) 364.

[3] G.K. Zipf, The Psycho-Biology of Language, M.I.T. Press, Cambridge, 1965; Selected studies of the principle of relative frequency in language, Harvard Univ. Press, Cambridge, 1932; Human behaviour and the principle of least effort, Addison-Wesely, 1949.

[4] D.L. Gerlough and M.J. Huber, Traffic flow theory, National Research Council, Washington, D.C., 1975.

[5] M.H.R. Stanley, L.A.N. Amaral, S.V. Buldyrev, S. Havlin, H. Leschhorn, P. Maass, M.A. Salinger, H.E. Stanley, Nature, 379, (1996) 804; B.B. Mandelbrot, Fractals and scaling in finance, Springer-Verlag, Berlin, 1997; Y. Lee, L.A.N. Amaral, D. Canning, M. Meyer, H.E. Stanley, Phys. Rev. Lett. 81 (1998) 3275.

[6] D. Martin, Trans. Inst. Br. Geogr. (NS) 14 (1989) 90.

[7] P.M. Allen, Proc. Natn. Acad. Sci. U.S.A. 73 (1976) 665.

[8] S. Lawrence and C.L. Giles, Science 280 (1998) 98.

[9] S. Redner, Euro. Phys. J. B 4 (1998) 131.

[10] D.C. Roberts and D.L. Turcotte, Fractals 6 (1998) 351.

[11] Note that if two or more family names are of same size, then we assign the same number for their rank and skip the next available numbers by the number of degeneracy and assign the new number for the rank of the next biggest family.

[12] D. Stauffer, Introduction to Percolation Theory, Taylor and Francis, London, 1985; A. 
Bunde and S. Havlin (eds.), Fractals and Disordered Systems, 2nd Ed., Springer, New York, 1996.

[13] P. Bak, C. Tang and K. Wiesenfeld, Phys. Rev. Lett. 69 (1992) 1629.

[14] A. Cohen, R.N. Mantegna and S. Havlin, Fractals 5 (1997) 95.

[15] M. Takemitsu, Myoji to Nihonjin (Family Name and Japanese), Bungeishunju Ltd., Tokyo, 1998. 


\section{FIGURES}

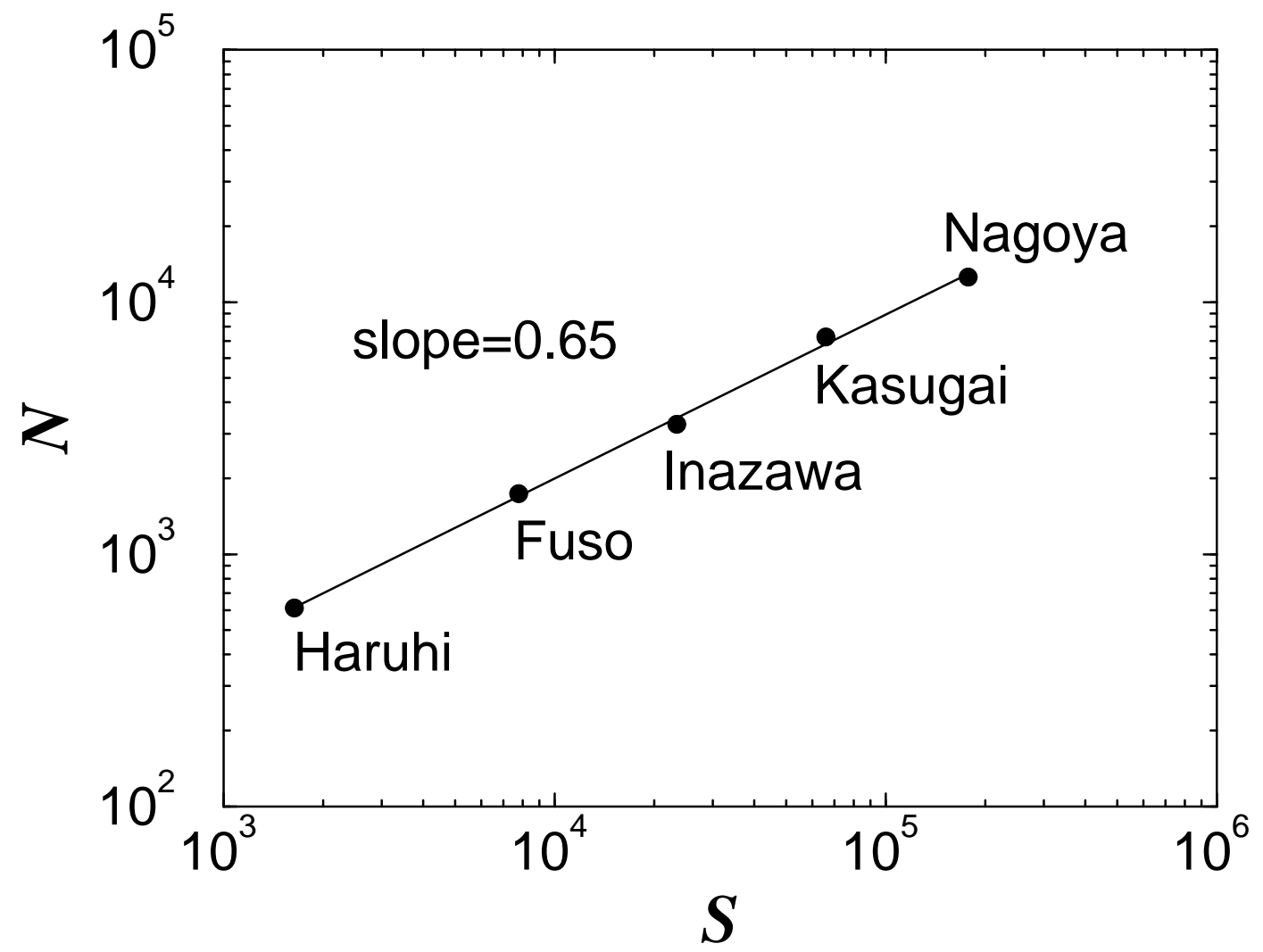

FIG. 1. The number of family names $N$ against the total population $S$ for five different regional societies in Japan shows power-law behaviour as $N \sim S^{\chi}$. By linear regression in double logarithmic plot we estimate the exponent $\chi=0.65 \pm 0.03$. 

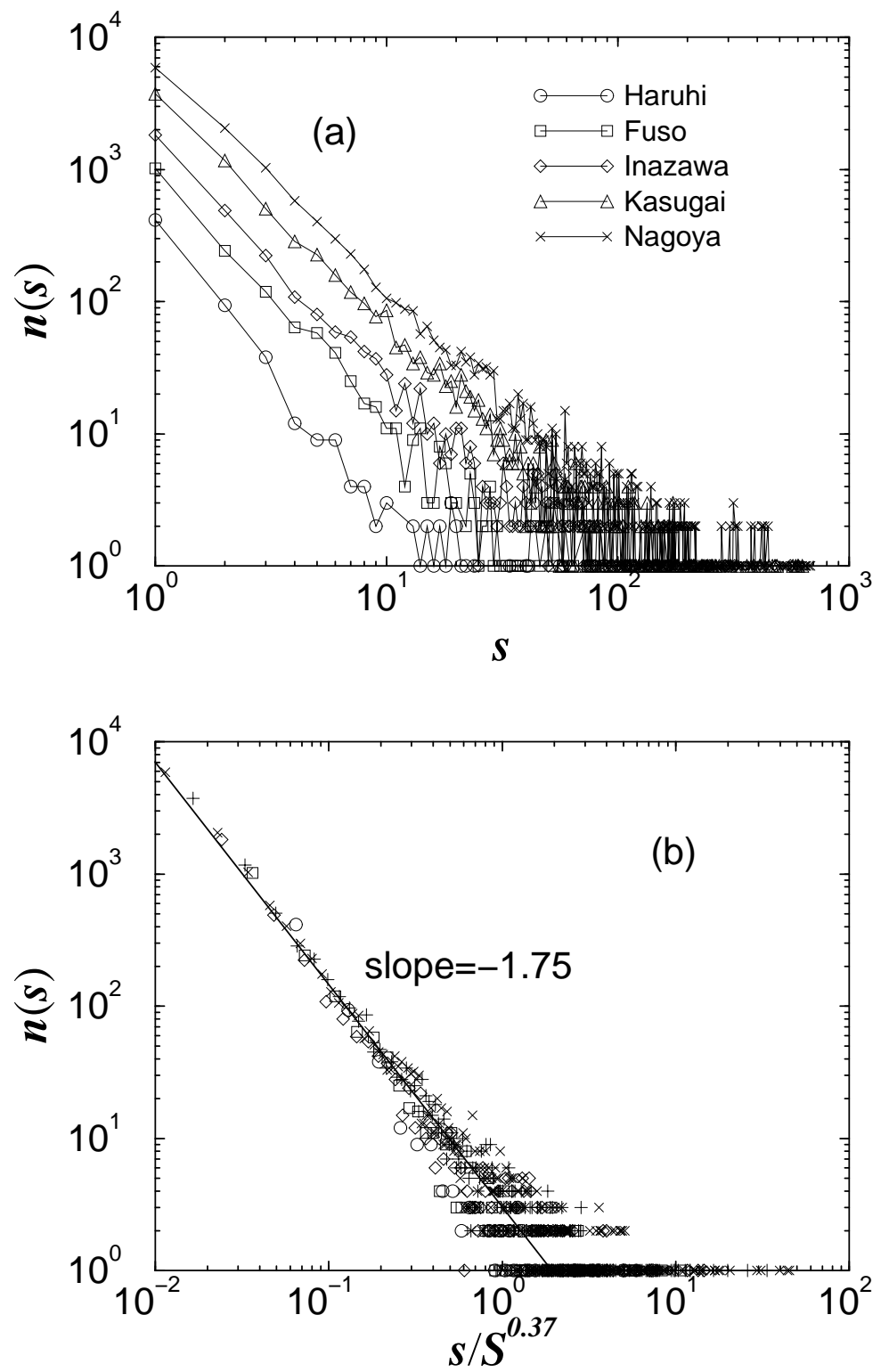

FIG. 2. a) The double logarithmic plot of the histogram $n(s)$ vesus family size $s$ for five regions in Japan. b) Data collapse using the scaling form in Eq. (2). The linear fit of the power-regime gives $\tau=1.75 \pm 0.05$. 

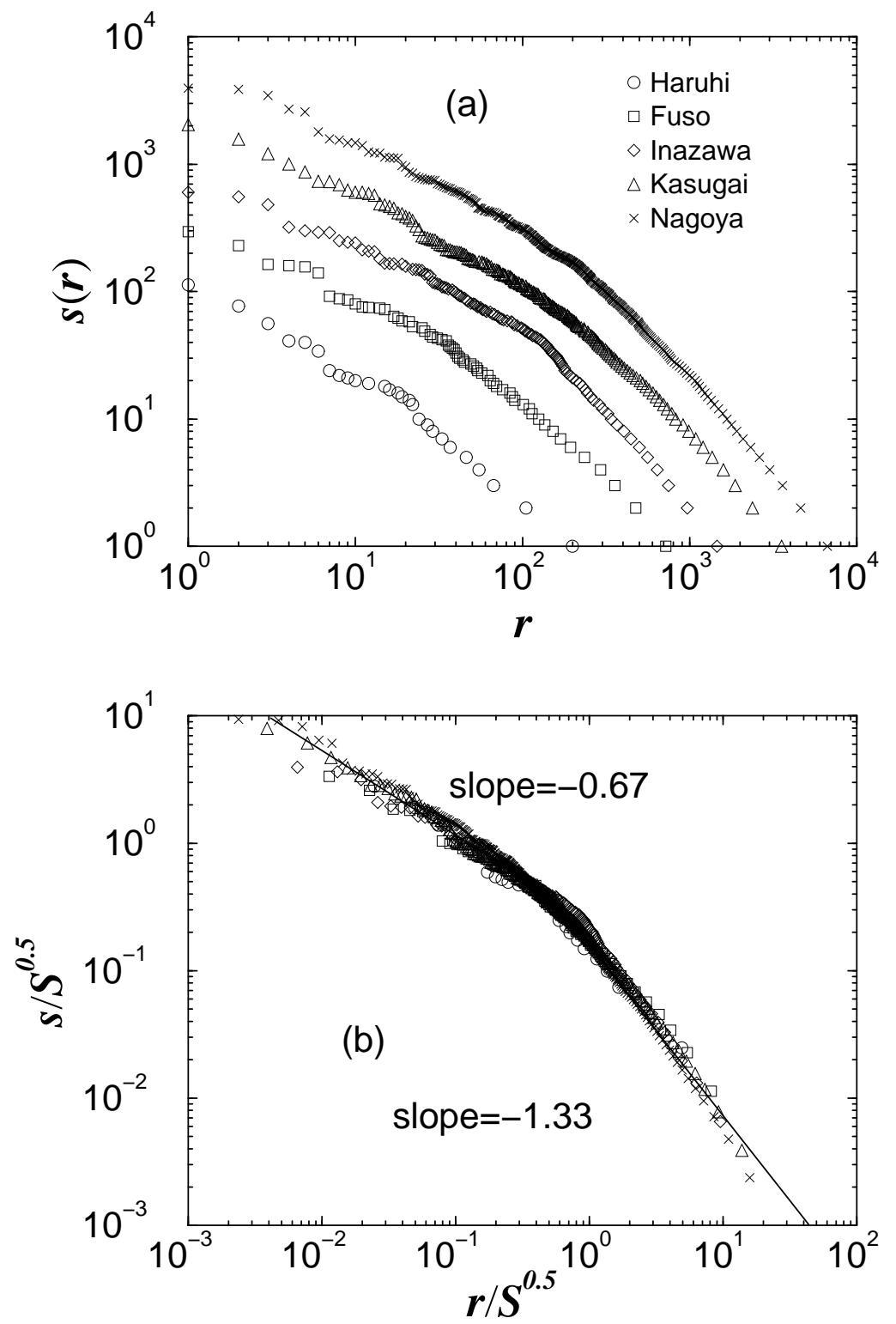

FIG. 3. a) The size of a family name $s(r)$ when plotted against the rank of the family name $r$ shows a crossover behaviour at the characteristic rank $r^{*}$ where $r^{*}$ scales as $S^{\alpha^{\prime}}$. The solid line connects the crossover points whose slope is one implying $s\left(r^{*}\right) \sim r^{*}$. b) Data collapse using the scaling form in Eq. (何). A crossover behaviour is observed from the $\phi_{I}=0.67 \pm 0.03$ to $\phi_{I I}=1.33 \pm 0.03$. 

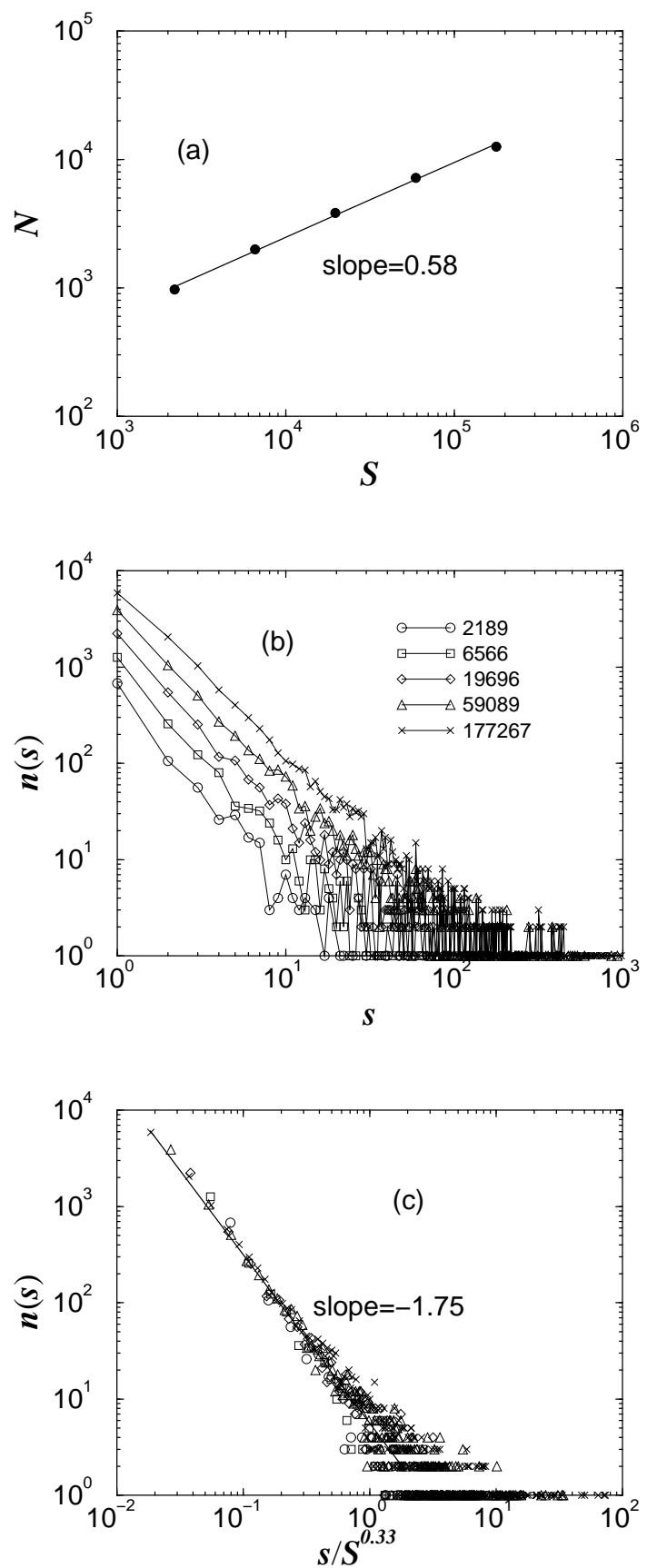

FIG. 4. a) The double logarithmic plot of $N$ versus $S$ obtained from the distributions for the randomly chosen population $S=2189,6566,19696,59089$ and 177267 . It shows a simple power-law relation, $N \sim S^{\chi}$ with $\chi=0.58$. b) The double logarithmic plot of the number of family names of same size $s, n(s)$, versus the size $s$. c) Data collapse using the scaling form in Eq. (2). The linear fit of the power-regime gives $\tau=1.75$. 